beau temps durable pendant presque tout le mois de junvier. - Une dépression se produit dans la première quinzaine de févicr, avec chutes de neige. La deuxième quinzaine est belle. Remarquer la corrélation avec les vents dominants de Nord-Ouest à Nord-Est, " ce qui esl normal en janvier et en févier " (Marciand, directeur de l'obscrvaloire du Pic du Midi, Bulletin de la Société Ramond, rgo8).

$3^{\circ}$ Malgré la courbe cxceptionnelle (hès haule) des quatre premiers mois de l'hiver 19o-igos, on peut établir que l'allitude moyenne de la neige sur le versant Nord des Pyrénées esl vers 1400 mètres cn novembre et vers 1200 mètres en décembre;

$4^{\circ}$ A parlir du milieu davril la fusion des neiges devient régulière, par suile de l'équilibre de la température et de l'accroissement graduel des heures d'ensoleillement;

$5^{\circ}$ Les fuisions les plus brusques sont dues au vent du Sud (Siroco), presque toujours très sec cl très cha'ud, ou au vent du Sud-Ouest, " amenant des averses très fortes el toujours locales "(Marchand).

\section{LA VOIE D'EAU DE MILAN A VENISE}

Jusqu'à ces dernières années, le réseau ilalien de navigalion intéricure élait très modestc, comportant un petit nombre de canaux pour la plupart devenus insuffisants devant les exigences de l'industrie moderne. La renaissance économique de l'llalie, si sensible ces dernières années, a provof'ué un vaste mouvement de l'opinion publique vers la création d'un réscau de navigalion intéricure répondant aux besoins de l'industrie et du commerce italicns, et leur permollant de recueillir les avanlages que de bonnes voies navigables procurent aux autres nations curopéennes.

Au dernier Congrès de navigation intérieure lenu à Namles les 6 , 17 el 18 juin 19 43 , M. Maro Berreta, secrétaire du Comilé pour la navigation intérieure à Milan, a présenté un remarquable rapporl sur lin "voic d'eau de Milan à Venise ", oì il monlpe l'intérêt que présenterail ce grand ourrage pour la rjche région lombarde. Nous extrayons de co lravail les parlies les plus essentielles, le projel résultant los dernières études de la Commission.

Dans ce projel, la roie d'rale serail constituce dabod par un camal élusé de Milan à Pizzighellone, sur l'Adda, puis par l'Adła canalisé sur i $\&$ kilomètres jusqu'à son conlluent avec le P'ó, par le Pó jusqu'au commencement du Molla, enfin par un canal du Pò à la lagune de Venise.

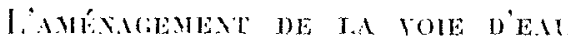

a). L Le canal de Milan à Pizigheltone (Adda) a un laas donl la longueur dépasse de $2 \%$ à peine la distance en ligne droite entre les deux extrémités.

Une première section de Milan à Cavenago 36 kilomelres environ) qui est exclusivement navigable, a une section transversale avec tahs d'mne inclinaison de $45 \%$, une lat geux au plafond de $22^{\mathrm{m}} 50$, de $28^{\mathrm{m}} 90$ cntre les bords, une profondeur lotale de $3^{\mathrm{m}}$ ?o, $\mathrm{m}$ movillage de $2^{\mathrm{m}} 50$ arec une largeur de $27^{m}$ bo it la flollaison, el une surface de section monillée de $62 \mathrm{mq}$. 5o. Elle est revêtue au plafond d'un béton de ciment lisse de $0^{\mathrm{m}} 30$ d'épaissenu ; sur les parois, la couche de bélon a une épaisscur qui va en décroissant de $0^{\mathrm{m}} 30$ a $0^{\mathrm{m}} 25$. Enfin, le canal est flanqué Jalóralement de deux ehemins de halage diune largeur de 5 màtres.

lne deuxieme section de Cavenago ì Pizzigheltone (16 km. a), qui ne sert pas exclusirement à la navigation mais qui est également utilisée pour la création d'énergie hydraulique. Elle a une section avce risberme de ${ }^{m} 30$; une largeur au plafond de $25 \mathrm{~m}$; une profondeur de $5^{\mathrm{m}} 20$ el un mouillage variant cntre $3^{\mathrm{m}}$ bo et $4^{\mathrm{m}} 50$. Sa section mouillé a par conséquent une grande surface. Le revêtcment est lo même que pour l'autre seclion.

Pour racheter l'importante différence de niveau existant entre le commencement el la fin de la $\mathrm{r}^{\mathrm{ra}}$ section du canal, $47 \mathrm{~m}$. environ, on a disposé le long du parcours dix écluses à sas, de type et à chute divers selon la pente plus ou moins grande du terrain traversé et de façon à avoir une distribution logique et avantagcuse des écluses; écluses in parlio avec des bassins d'épargne (40\% d'épargne) et chute de $5^{\mathrm{m}} / 45$, et en partic avec chutc de $3^{\mathrm{m}} 25$. Toutes ces écluses onl les dimensions utiles de jo $\mathrm{m} . \times$ ro $\mathrm{m}$.

Dans la deuxième section, sont disposées deux écluses a sas, latéralement à deux centrales hydroélectriques destinéns à utiliser l'eau dérivée de l'Adda par un barrage de prise à Cavenago, auquel fait suite un canal secondaire de dérivation qui se jette dans le canal principal.

La puissance effective qui peut elre fournie par ces deux installations est de g ooo $\mathrm{HP}$, pendant le jour, moindre pendant la nuit et pendant les périodes de basses eaux (ninimum nocturne 3 ooo $\mathrm{HP}$ ) en utilisant comme réservoir d'eau, pendant les heures où la consommation d'énergie diminue, la section de canal en amont jusqu'à la dérivation, un bras mort de l'Adda el la section du fleuve en amont de la dérivation que l'on a projeté avec barrage.

La hauteur de la première chule utilisable variera de $8^{\prime \prime} 6$. à $7^{\mathrm{m}} 7^{5}$, selon lo niveau de l'eau dans le réservoir de Cavenago ; la seconde scra également variable, selon le niveau de l'cau dans l'Adda el s'approchera de $7^{\mathrm{m}} 7 \mathrm{o}$.

De soigneuses mesures du débit de l'Adda, ont amenć à conclure que le débit sùrement disponible est de 32 mètres cubes pendant to mois de l'annće. Grâce à l'emmagasinage projelé qui est de 850.000 mètres cubes sur une différence de nivcau de $o^{\mathrm{m}} \mathrm{go}$, on pense obtenir sưrement la quantité d'énergie précitéc.

La vilesse de l'eau dans le canal navigable entre Milan cl Carcnago, sera théoriquement mulle ; dans le canal navigable industricl entre Cavenago et Pizzighettone, elle sera de $0^{m} 50$ cnviron par seconde, la section et la pente ayant été calculées de façor à entraìner à celle vitesse les fo mètres cubes de son débit normal dans les sections en amont, et de 70 mètres cubes dans Jes seclions on aval.

Comme on l'a dit, le canal projeté sera construit avec un revêtement étanche robuste, de facon à constituer une sorte d'énorme vase qui, en aucum point, n'ajoutera ni n'enlevira de l'cau au sous-sol du terriloire traversé ; pour la navigalion, il aura cnsuile te grand avantage d'empêcher la végilation el préscntera, par conséquent, moins de résistance au mouvement des batcaux.

On élimine ainsi les perturbations occasionnécs trop sonrenl par l'ouverture des grands canaux dépourvus de rer: tement élanches qui peurent produire dans quelques zonos plus ou moins étendues un abaissement du niveau des caux souterraines, au grand dommage des puits d'eau polable ti du débit des sources servant à l'irrigation. Dans d'autres ondroits, au contraire, on pouvait craindre une élévation du niveau des caux soutorraines, nuisible pour les habitations of la pureté des eaux potables dans les puits, la formation de marćcages, on même seulement la diminulion de la produclion agricole, par suile di rapprochement excessif de la nappe souterraine par rapport à la surface du sol. Ces inconvénients auraient pu ensuite donner licu à des procès 
pour dommages-intérêts el aux Lravaux nécessaires pour les ćliminer et, en tout cas, à de dangereuses conséquences pour l'hygiène el la production de la zone traverséc. Le revêtement étanche projeté, suivant les conclusions du Comilé Promoleur dissipe tout doute en ce qui concerne la possibilité de perturbations du régime actuel des eaux souterraines.

Les frais de constraction des deux canaux Milan-Cavenago, Cavenago-Pizzighettone sont estimés à $4 \mathrm{x}, 5$ millions de francs, ce qui donne (déduction faite de 9 ooo ooo de francs, valcur de l'énergie créée) environ 625 ooo $\mathrm{fr}$. au kilomètre.

b) La section de Pizzigheltone au Pô. - Pour la section de Pizzighettone au Pó, la Commission ministérielle projetait de suivre le cours libre de l'Adda, contrairement à ce qui avait été proposé par le Comité de Milan pour la Navigation intérieure qui prévoyait qu'il serait très difficile d'obtenir en courant libre les mouillages suffisants et proposait par conséquent soit la canalisation, soit la continuation d'un canal jusqu'à Crémone.

Des études ultérieures très séricuses ont fail conclure à l'opportunité de choisir une des solulions proposées par le Comilé de Milan : Le canal Pizzigheltone-Crémone, d'une longueur de r 8 kilomètres, coûterait 7 ooo ooo de francs et aurait deux écluses. La canalisation de l'Adda coûlcrait 3500 ooo lires, comprenant trois écluses de convois, d'une longueur de $\mathrm{x} 65 \mathrm{~m}$. et d'une largeur de ro $\mathrm{m}$. et des travaux de régularisation des bergés et du lit.

Le canal Pizzighettone-Crémone en abrégeant de 20 kilomètres le parcours entre Venise et Milan, constituerait en outre la première section d'un grand canal entre Crémone et Mantoue qui aurait une grande importance, spécialement en ce qui concerne les communications entre Milan et le Lac de Garde.

Ce dernier canal est particulièrement appuyé par la ville de Mantoue, mais sa construction est évidemment moins urgente que les autres travaux et est renvoyée à une seconde phase du programme d'exéculion des voies d'eaux dans la vallée du Pô.

c) La voie d'eau du Pô. - La largeur du fleuve au con. fluent de l'Adda est de 288 mètres, elle atteint 320 à 350 mètres dans les régions Crémonaise el Mantouane.

Sa navigalion est possible pendant près de 330 jour's par an, mais elle est gênée par des seuils en plusieurs poinls du 1lcuvc. Ces grèves se produisent dans les courbes où le courant de relour dépose les matériaux arrachés à Ja berge attaquée directement, mais surtout en aval du confluent des torrents de la rive droite, dont les caux très limoncuses abandonnent les malières enlevées aux pentes dénudées de l'Appenin, et en amont du confluent des affluents de la rive gauche dont le débit considérable cause une diminution de vilesse du Pó favorisant le dépòt des sables.

On peul diviser colle parlie de la rivière en deux seclions : la $I^{r \theta}$ de 205 kilonètres de l'embouchure au conflunnt de l'Oglio présente une différence de niveau de 55 mèlres, la $2^{e}$ de rra kilomètres du confhuent de l'Oglio à celui de l'Adda, 35 mètres.

a) Dans la première seclion (205 kilomèlres, de l'Oglio à l'embouchure) on a toujours des mouillages. supérieurs à deux mètres, excepté sur trois scuils d'une longueur tolale de $\mathrm{r} .320$ mòtres qui alleignent une hauteur de $0^{\mathrm{m}} / 47$ a $\cdot \mathrm{I}^{\mathrm{m}} 20$ au-dessus de la ligne des mouillages de $2 \mathrm{~m}$, de sorle qu'ils laissent un mouillage minimum de $o^{\text {ng }}$ o.

b) Dans la scconde section (1 12 kilomètres, de l'Oglio à
l'Adda), on a pendant loule l'année 2 m. el mène davanlage, excepté sur 27 seuils d'une longueur lotale de $11.862 \mathrm{~m}$. el d'une hauleur de $0^{m}{ }^{6} 6$ à $x^{m} 40$ arec un mouillage minimum de $o^{m} 60$.

Ces conditions ne sonl pas défarorables, fuand on punse: qu'elles résultent d'observations failes pendant une période d'annćes où aucun travail de régularisalion ou de dragage, n'avait été commencé.

Elles permettraient à un balcau de 600 lommes $65 \times 8$ $\times x, 75)$ de naviguer jusqu'au confluent de l'Adat $(3-7$ kilomètres) dans les conditions suivantes :

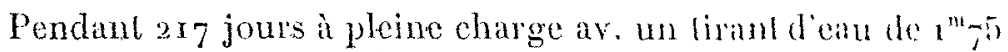
- $74-\mathrm{a} 1 / 2$ charge - - - $1^{\mathrm{m}} 20$

- 39 - avec $x / 4$ de charge - - $0^{m} 80$

Soit : 330 jours de navigation à diverses charges.

Ces condilions peuvent bien être comparces à celles dans lesquelles se trouvaient les grands fleuves allemands avant. les grands travaux récents.

Mais il faut faire les travaux nécessaires à la régularisalion du fleuve; le barrage des bras scendaires, de manièe a concentrer toules les caux basses dans le bras principal ; la fixation des rives suivant un tracé rationnel, el au moyen de digues longitudinales el d'épis plongeants, la plantation et la fixation des grèves ef des bancs, elc.

Ces travaux devront èlre accomplis suivant une haule unité de vucs et suivant un plan rationnel, qui doit ctre élabli après une élude séricuse des élóments conslitulifs du fleuve el de leur régime, el après des cxpéricnces réilércés de régularisalion des diverses sections. Ces travaux devionl, cnsuite être complétés par l'installation d'un service régwicr de dragage destinć à l'entrelien du chenal.

Le Gouverinement italicn a nommé une Commission ponn le régime du Pó sous la présidence de M. Romanin el a tuis à la disposition du "Compartiment du Pò n du Génic Civil, trois dragues avec toul le matérid nécessanire pour les expériences de dragage.

Ces expéricnces se font dojà depuis quelques anmés aroc le plus grand soin, mais on recommaîl qu'il est indispensable de mettre à la disposition de celle station spécialc, un malé. riel bien plus complet et qu'on commence immédiatement les études el les travaux pour la régularisalion du lit do loule la section du Pó qui va du confluent du Tessin à Gavanclla sans l'exécution desquels te dragago intermillont ne peut donner les résultals nécessaires.

Les résultals anxquels la régularisalion doil lendre, sulivani les conclusions de la Commission ministórielle perr la Navigation intérieure, scraicnt d'oblonir jusfu'au conllusul du Tessin un mouillage de 2 metres pendant loule l'auméc

d). Les Canaux du Pò à la mer. - L'embouchuro da Pó. fleuve qui n'a pas un vaste estuaire, mais de nombreuses bouches formant delta, subit un vérilable étranglement, i cause des bares qui rendent la navigation des grands bateaux presque impossible. Le Pó n'a pas pu, par conséquent, dans son cours inférieur. (qui pendant plusieurs kilomèlros a des mouillages assez profonds pour permethe la navigation même à des bateaux de mer) être utilisé comme les grands fleuves dir nord.

Les ports de l'Adriatique ont da se eréer bien loin dos bouches du Pô, pour ne pas voir leurs bassins ensablés par les matériaux que le fleuve transporle.

Sur un avant projet de M: Barcelloni Corte, ingénieur du Génie Civil, on a dressé le projet d'un grand canal entre 
l'Adige et le Pó, et l'élargissemet de la ligne actuclle entre l'Adige et la lagune.

la section adopléc pour celte ligne cst de $20 \mathrm{~m}$. au plafond, de $36 \mathrm{~m}$. au bord des berges, ce qui donne une largeur de $30 \mathrm{~m}$. à Ia flottaison avec un mouillage de $2^{\mathrm{m}} 50$ (eaux basses) et de 36 mètres avec un mouillage de 4. mètres (eaux moyennes).

Ces canaux n'ont pas de différences de niveau à vaincre, mais ils ont cependant des écluses à leur débouché dans le Pó, dans l'Adige et dans le Brenta. Ces ćcluses resteront ouverles la plus grande partie de l'année. Elles ont une longueur utile de $j^{\circ} \mathrm{m}$. of une largeur utile de $9 \mathrm{~m}$. Il est à prevoir que dans un projet d'exéculion ces écluses auront des dimensions bien plus considérables, de façon à donner passage aux cargo-boals et aux convois de chalands remorqués.

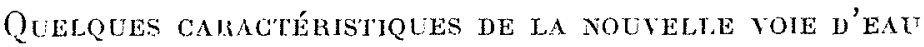

La nouvclle voie navigable qui sera créée entre Venise et Milan aura une longueur de 38 kilomètres qui pourra chre réduite a 360 kilomètres par le canal Pizzighettone-Crómone et a 340 par le canal Crémone-Mantoue.

La durée de la navigalion, élant donné que le phénomène des glaces n'est pas à craindre, peut être évaluée à 330 jour's comme il a été indiqué plus haut.

Les mouillages auront un minimum de $2^{\mathrm{m}} 50$ dans les canaux; en plusieurs sections ils iront jusqu'à $4 \mathrm{~m}$. Sur ic Pó, on a vu que jusqu'au confluent de l'Adda, ils ont aclucllement un minimum de $2 \mathrm{~m}$. pendant $2 \mathrm{I} 7$ jours; après les travaux de régularisation ce minimum sera certainement alteint pendant tous les 330 jours.

Le profil en long de la voie d'eau est excellent. Dans la section qui a élé étudiée, le Pó a une pente plus dóuce que le Rhin. Le conlluent de l'Adda, point le plus en amont de la section du fleuve comprise dans celte ligne, qui est à une distance de 3r/4 kilomètres de l'embouchure, a unc altitude (pendant les eaux moyennes) de 36. mètres au-dessus du niveau de la mer.

Les canaux entre la Lagune et le Pò n'ont pas de difrérences de nivcau à vaincre; celui cntre Milan of le Pó no rencontre sur un parcours de 72 kilomètres qu'une différence de niveau de $66 \mathrm{~m}$. environ avec 15 écluses qui n’ont pas des chutes excessives.

La seclion trensversale des canaux se prête dans des conditions pleinement satisfaisantes ì la navigalion des bateaux de 600 tonnes.

En effel, la valeur du rapporl entre Ia scelion moulléc su cantil et li section monilléc des batenux types de 600 tonnes col Loujours supérjeure à 4 , ce qui permettra de réaliser de bonnes vitesses mème sur les canaux.

Elant donné les conditions favorables quui ont été cxposćes, une navigation très puissante pourra avoir licu sur la nouvelle voic. Et on peut croire que des bateaux d'un tonnage supérieur à 800 tonnes pourront naviguer presque pendant toute l'année, jusqu'au confluent du Mincio.

Dans le cas où la ligne du lac de Garde au Pô, par Mantoue et le Mincio, serait également étudiće avec une grande largeur de vues, c'est-à-dire que le projet comporte des canaux à grande section el des écluses à grandes dimensions. celle navigation, avec des bateaux de 600 , de 800 et peut-être de rooo tonnes pourra s'étendre jusqu'au lac de Garde at jusqu'aux territoires de l'cmpire d'Autriche-IIongrie.

Pour les transports destinés ou provenant des ports silués en amont du confluent du Mincio, la navigation pourra être excrcée au moyen de bateaux de 600 tonnes jusqu'à Milan.
Etant donné les dimensions des canaux entre le Pó el la Lagune, des cargo-boats pourront, comme entre Londres et Paris, faire un service direct entre les ports fluviaux du Pò et les ports de l'Adriatique.

La traction sera exercée, en Lagune, comme on le l'ait aujourd'hui, au moyen de remorqueurs à vapeur à hólice qui" traineront des convois de 2 ou 3 chalands. Il en sera de même, - sans danger pour lo plafond et les talus, étant donné les dimensions de la section transversale - sur les canaux reliant le $P o ̂$ et la -Lagune et sur le canal depuis Pizzi, Ghetone, jusqu'à Cavenago d'Adda, ainsi que sur l'Adda canalisée.

Sur le canal entre Cavenago d'Adda et Milan $(36 \mathrm{~km}$.$) , on$ prévoit au contraire l'installation de la traction électrique individuelle avec tracteur sur berges.

On prévoit l'emploi sur le Pồ de remorqueurs à roues d'une puissance de 500 à 600 HP qui pourront remorquer en remontant le courant à la vitesse de 4 à 5 kilomètres à l'heure et à la vitesse de 8 à r 2 kilomètres en le descendant, des convois de trois bateaux de 600 tonnes.

Ce sont là des condilions bien meilleures que celles du Rhône, où, pour des convois semblables, la Sociélé de remorquage récemment fondée, a fait construire des remorqueurs d'une puissance de r.200 à X.800 HP.

\section{GondtTIONS Futures d'ExplotTation}

Quelles pourront être les conditions d'exploitation et Jes prix de transport de la future navigation entre Venise et Milan?

Pour répondre à celle question, l'auteur, M. Mario BEIETT'A, a fait de soigneuses études el des calculs très minutieux (suivant les systèmes désormais rendus classiques parles remarquables études de Sympher) pour analyser tous Ies éléments constitutifs qui entrent en jeu dans la formalioil du prix de revienl du transporl par voie d'eau:

Ces éléments ont élé éludiés dans la pratique des Sociétés de navigation fluviale italiennes, et on a ensuite recherché quelle influence exercerait sur eux l'amélioration des condilions de navigabilité des voies d'eau existantes, l'application de systèmes modernes de traction et la création de nouveaux grands canaux.

L'analyse et la comparaison avec les mêmes éléments d'exploitation de la navigation sur les grandes voies modernes de la France, de la Belgirque et surtout de l'Allemagne; a permis de faire des prévisions qui, étant donné la sćvérité de la méthode suivie, ne dojvent pas être trop éloignées de ce que scront les conditions d'cxploitation.

En ce qui concerne le prix de revient du transport, M. Berelta cite un exemple relatif au parcours Venise-Milan :

I $^{\circ}$ Etant donné que le Pô, dans les condilions actuellis, permet, pendant une durée de navigation de 33o jours par an. à des balcaux de 600 tonnes :

$$
\begin{aligned}
& \text { Une immersion de } x^{\text {th }} 75 \text { pendant a } 77 \text { jours }
\end{aligned}
$$

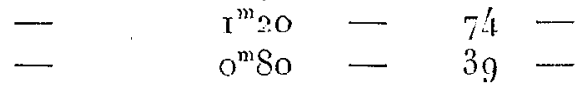

et supposant qu'on emploie :

\section{Le remorquage à vapeur :}

a) Pondant $27 \mathrm{~km} .5$ sur la lagune, avec remorqueurs à une hélice de roo à $\mathrm{r} 50 \mathrm{HP}$ et convois de 2 baleaux;

b) Pendant $25_{7}$ kilomètres sur le Pô avec remorqueurs fluviaux à roues et convois de 3 bateaux ;

c) Pendant ig kilomètres sur l'Adda canalisée; pendant $24 \mathrm{~km} .5$ sur les canaux de la lagune au Pô, avec remor- 
queurs à deux hélices d'une puissance de so à $10011 \mathrm{P}$ et des convois de 2 baleaux;

La lraction électrique (avec locomolrices sur rails) pendant 52 kilomelues, de Pizzighellone ì Milan ;

Supposant sur la ligne entière un trafic de? millions de tomnes, dont cing sixiemes en montére rers Milan el un sixième descendant sur Vonise;

Elanl donné pour des batcaux de $600 \mathrm{~T}$. un chargement:

Dans les voyages en amonl :

Pendant 2y jours de 600 lonues (immersion de $1^{\mathrm{m}} 75$ )

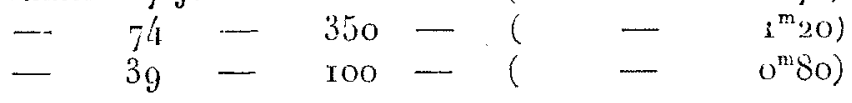

Dans les voyages en aval:

Pendant 330 jours de 120 tonnes (immersion de o ${ }^{\mathrm{m}} 65$ )

Supposant la vilesse moyenne de marche:

De 4 à 5 kilomètres daus le voyage cn amont sur toul le parcours el dans le voyage en aval sur les r oo kilomètres de lagune, de canaux el de l'Adda canalisée ;

De 8 kilomères dans le voyage en aval pour les $257 \mathrm{kilo-}$ mèlres sur le Pó;

En calculant que le parcours joxtrnalier pout. une moyemno de $x 3$ heures de navigation diurne est de 60 hilomèlres pour le voyage en amont et pour le royage en arial sur les camar, la lagune el l'Adda canalisée; de ró kilomèlres pour les royages en aval sur le Pô;

Considérant que la longueur j'éelle du parcours VeniseWilan est de 380 kilomètres, et la longueur virtuelle, à laquelle sont appliquées les bases du tarif calculécs suivan! les allongemenis habiluels de parcours, pour lemps perdu au passage des écluses, est de $434 \mathrm{~km}$. 5;

Le prix de revient du transporl d'une lonne de marchandises lourdes chargées en vrac, de Venise à Milan (y compris Ios charges terminales, exception-faile du déchargement, y compris les amorlissements, la manulention, Je personne!, les assurances, un pourcenlage de revenu industriel, escompté le tomps perdu pour la formation el la dislocation des convois, Jeur altente, la (raclion, elc...) a ćlé largement évalué à L. 6,7o environ, ce qui donnerait un coût unilaire de I, c. 7 par chaque tonne-kilomètre réelle et $r$ c. 5 par chaque tonne-kilomètre virtuelle.

$2^{\circ}$. L Ligne à polentialilé complèle. -- Lorsque le pó aura son chenal rógularisé de façon à oblenir gue pendant unc durée de navigation de 330 jours on ail loujours une immersion de $r^{\mathrm{m}} 75$ pour le baleau de 600 tonnes;

Considérant les mêmes circonstances énoncées dans le cas précédent et un trafic égal de doux millions de tonnes, donl les $5 / 6$ en montée vers Milan ct $1 / 6$ en descente sur Venise, le prix de revient du transport d'une tonne de Venise à Milan, y compris les charges terminales, s'éleverait à L. 4,95 et lo prix de revient unitaire respectivement à $L$. o, or 3 et I. o,or I. Gette diminution est obtenue seulement par la meilleure ulilisation du matéricl et tout en ne tenant pus compte de l'avantage que ressentiraient beaucoup des éléments qui constituent le prix de revient du transport, étant donné les meilleures conditions de navigation du Pô.

Co prix de revient est calculé largement et lescendrait cncore avec l'augmentation du trafic (augmentation qu'on poul próvoir comme sûre, étant donnée la polentialité des travaux artificicls de la ligne et le développement économique des régions traversécs) mais il a déjà mo valour intrinsòrue remarquable de bon marché.

Il serail trop long de résumer lous les avantages que procurera la nowvelle ligne, capable de permothe un transport aussi bon marché de grandes quanlités de malrchandises encombrantes et peutétre même des services a grande vilesse pour d'aulres marehandises de plus de valeur.

On peul bien se les imaginer quand on solnge a lagratidissement de la sphere d'influence du port de Venise, qui actuellement n'est pas utilisé élant domé qu'il n'a pas d'hinterland pourvu de roies d'eau modernes, - el an contact direct des régions industriclles du Milanais of des plaines agricoles de la basse vallée da Po, arce lous les pert. des colles de l'Adrialique el de la péninsule des Balkans, dont le port de Venise est l'escale naturelle pour l'llalie aptentrionale.

Les industries Jombardes ont besoin de millions de lonnus de malières premières qui leur arrirent exclusirmonl jar voic ferrée à dos prix tròs clevés.

l'conomie sur le prix de hanspont occasionnelat un dive-

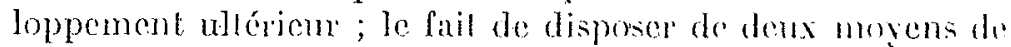
transport indépendants l'un de l'inulre, remdria les cohanger plus sûrs et plus rapides.

La nouvelle voie aura également une grande influence sur. les condilions du port de Gênes, où actuellement le trali: étoulfe par suite de l'insuffisanec des moyens de póncilation, mais qui, dóbarrassé du pénible service des chatbne, pourra donner tous ses cfforts anx trafies plus remmuét:leurs qu'il a perdus pendant ees dernières amécs, el pourra même devenir (ce à quoi il aspire depuis Jongtemps aver Marseille) un port d'approvisionnoment de la Suisse commt. Jes ports du nord de l'Europe.

On n'ignore pas on Italie les avanlages qu'on pelireronl lis chemins de fer, parce qu'étant débarrassés du transport des marchandises pauves et du service onéreux des charbons dans le port de Gênes (qui leur impose d'envoyer, à l'aller, des wagons à peu près vides), ils pourronl ulidiser leur orga. nisalion d'une façon plus intense pour le transport de mar. chandises riches ot éviler ces crises de transport qui sont actuellement si préjudiciables au développenent des indıstrics lombardes.

\section{VOIES NAVIGABLES ET HOUILLE BLANCIIE}

Nos lecteurs auront remarqué avec quelle attention nous suivons, dans cette Revue, toules les questions relalives au développement de la navigation intérieure. Cost parce que nous sommes intimement convaincus que l'aménagement de grandes voies de transport par eau, dans nos régions de houille blanche, doit accroître cn de très grandes proporlions l'activité industrielle de ces régions.

Il faut en effet rélléchir aux considérations suivanles : Dans les régions industrielles du Nord, où Jes canaux sonb le plus développés, ils constituent un outillage de premièce importance pour l'apport des combustibles et des malieres premières áux usines qui se sont multipliées entre les mailles de leurs róseaux. Ici, la houille noire vient aux fours mólallurgiques, aux foyers des machines thermiques presque cxclusivement par ces voies d'eau; mais avec la houille y passent aussi les matieres pondéreuses qui sont transformécs par nos grandes industries du Nord et de l'Fst. Le lonnage des marchandises ainsi transporlé est considérable el juslific l'cxistence de la voie fluviale.

Dans les régions de houille blanche où le charbon manque, où n'existent pas encore d'industries absorbant de gros tonnages de matic̀res premières, ou enfin le sol accidenté no se prête pas à la créalion de vojes d'ean, il semble que celles- 